\title{
Control biológico del vector del nematodo de la madera del pino Monochamus galloprovincialis Olivier mediante autoinfección con el hongo entomopatógeno Beauveria pseudobassiana S.A. Rehner \& Humber
}

Self-dissemination of entomopathogenic fungus Beauveria pseudobassiana for biological control of Pine Wood Nematode vector

Monochamus galloprovincialis Olivier

Sacristán, A. .*, Fernández-Bravo, M. ${ }^{2}$, Quesada-Moragas, E. ${ }^{2}$, Pajares, J. ${ }^{1}$

${ }^{1}$ Instituto universitario de Investigación en Gestión Forestal Sostenible. Universidad de Valladolid-INIA, Avda. de Madrid 50, 34004, Palencia, España

${ }^{2}$ Departamento de Agricultura y Ciencias Forestales, ETSIAM, Universidad de Córdoba, Campus de Rabanales, Bldg-C4 Celestino Mutis, 14071, Córdoba, España

*Autor para correspondencia: alberto.sacristan@forest.uva.es 


\section{Resumen}

Bursaphelenchus xylophilus, cuyo vector es Monochamus galloprovincialis, provoca la enfermedad del marchitamiento del pino. Las estrategias de control se centran en el manejo del vector.

En este trabajo se desarrolla un método de autodiseminación de Beuaveria pseudobassiana, hongo entomopatógeno de alta virulencia sobre $M$. galloprovincialis, que permita la infección sobre la población de este vector, consistente en un sistema mixto de autoliberación y autoinfección, de los insectos capturados en el bote colector de trampas multiembudos. Se intenta aumentar su selectividad reduciendo las capturas de especies de xilófagos no objetivo mediante modificaciones en el sistema: filtrado a través de mallas de fondo y alturas de conexión del dispositivo al bote. El sistema de malla ancha testado redujo en un $72 \%$ la captura de especies no objetivo. El dispositivo de autoliberación de Monochamus, un tubo enroscado externamente al bote colector, ha permitido la autoliberación de la totalidad de los insectos capturados.

Se han determinado las características del dispositivo de autoinfección y la concentración del hongo entomopatógeno que pueden resultar adecuadas tras ensayar dos concentraciones: $1 \cdot 10^{9}$ y $1 \cdot 10^{10}$ conidios gramo ${ }^{-1}$ en talco y dos dispositivos. El dispositivo estrecho forrado internamente con fieltro fino y la dosis de $1 \cdot 10^{9}$ conidios gramo ${ }^{-1}$ en talco han definido un tiempo promedio de vitalidad (4,9 días) y una cantidad de inóculo transmisible $\left(2,7 \mathrm{mg} \cdot\right.$ insecto $\left.^{-1}\right)$ adecuado.

Palabras clave: Dispositivo de autoinfección, Autodiseminación, Atrae e infecta, Enfermedad del Marchitamiento del Pino, Nematodo de la madera del pino, Bursaphelenchus xylophilus, conidios en talco, enfermedad de cuarentena.

\section{Summary}

Pine Wood Nematode Bursaphelenchus xylophilus causes Pine Wilt Disease (PWD), resulting in widespread tree mortality. This quarantine organism is necessarily vectored by Monochamus spp. pine sawyer beetles. PWD management through control of the insect vectors has been proposed. Recently, the high potential of entomopathogenic fungus Beauveria pseudobassiana for the biological control of M. galloprovincialis, the only known vector of the disease in Europe, has been shown.

Several experiments were carried out aimed to develop a practical method to spread this biological control agent into the beetle population by self-dissemination tactics. Multiple funnel trap collection cups were provided with screened bottoms to effectively reduce by-catch of non-targeted beetles by $72 \%$. Two self-release devices attached to the trap collection cups were field tested and resulted high level effective in allowing escape of trapped M. galloprovincialis adults. These devices were further developed to serve for the self-infection purpose. Two B. peudobassiana concentrations, $\left[10^{\circ}\right]$ and $\left[10^{10}\right]$ conidia/g in talc powder, were assayed in two selfinfection devices in the laboratory. Survival time averaged was 4,9 days and dosis $2,7 \mathrm{mg} \cdot$ insect $^{-1}$ was reported for the low dosage applied with thin device. Further studies testing horizontal transmission of self-infected beetles would extend these results.

Keywords: Self-infection device, Self-disemination, Lure \& Infect, Pine Wilt Disease, Pine Wood Nematode, Bursaphelenchus xylophilus, dry conidial, quarantine pests. 


\section{Introducción}

La comprobación de que Monochamus galloprovincialis Olivier (Coleoptera: Cerambycidae) actúa como vector del Nematodo de la Madera del Pino (NMP) en Portugal (Mota et al., 1999; Sousa et al., 2001) lo ha convertido en un insecto de relevante importancia fitosanitaria (Alvarez et al., 2016). El NMP (Bursaphelenchus xylophilus (Steiner and Buhrer) Nickle (Nematoda: Aphelenchoididae)), en cuarentena en la Unión Europea (Lista A2) (EPPO, 2016), causa la Enfermedad del Marchitamiento del Pino (EMP). Se aloja en los canales resiníferos de las coníferas susceptibles y se alimentan de las células epiteliales y parenquimáticas, lo que desencadena una respuesta del hospedante que conduce a la perturbación del transporte hídrico y su muerte rápida por cavitación (Fukuda, 1988; Hara et al., 2006).

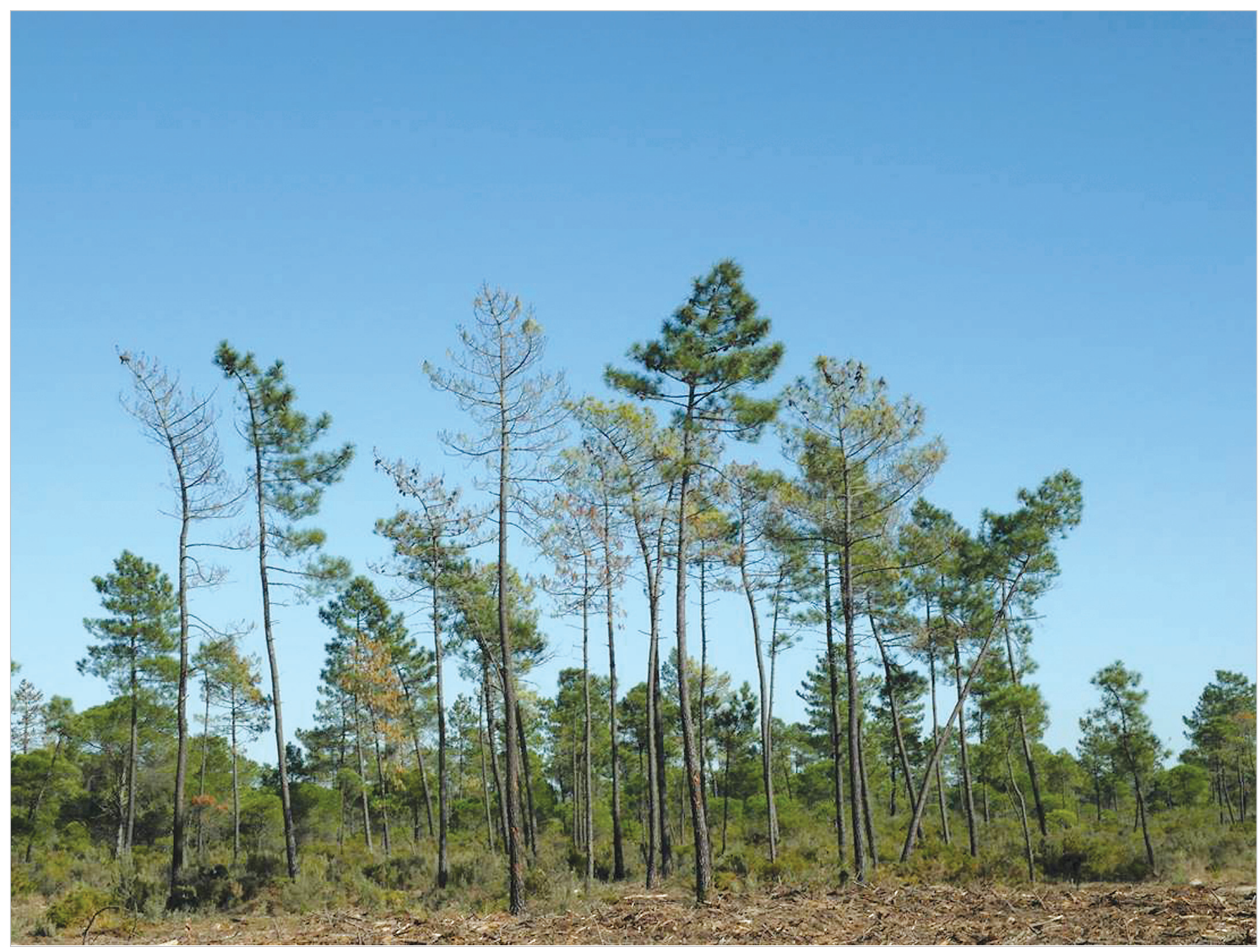

Figura 1. Rodal de Pinus pinaster afectado por la Enfermedad del Marchitamiento del Pino en Portugal. J. Pajares.

El control del NMP es complejo debido a las características biológicas, ciclo reproductivo corto, fases fitófagas y micófagas y resistencia de larvas dauer (Mas i Gisbert, 2015), así como su ubicación en el xilema. Por tanto, el control de la enfermedad se ha enfocado al manejo de su vector. 
El control de Monochamus sp. es una estrategia para frenar la expansión de la EMP. La disponibilidad comercial de un atrayente cairo-feromonal para su captura (Galloprotect-2D ${ }^{\circledR}$, SEDQ, Barcelona) (Pajares et al., 2010 y 2013; Alvarez et al., 2016) y la mejora de las trampas (Alvarez et al., 2015), han permitido un eficiente monitoreo de las poblaciones de Monochamus. La fracción cairomonal: ipsenol y metil-butenol (Pajares et al., 2004; Ibeas et al., 2007) es atractiva a diversos coleópteros que forman parte del hábitat subcortical de los pinos, efecto indeseado (Martín et al., 2013).

Entre los agentes biológicos, Naves et al. (2008) descubrieron el hongo Beauveria bassiana (Bals. Criv.) Vuill., causante de la mayor mortalidad en M. galloprovincialis. Shimazu (2004) y Maehara et al. (2007) han realizado ensayos sobre $M$. alternatus con este hongo. Alvarez-Baz et al. (2015) demostraron el potencial entomopatógeno de la cepa EABpb de B. pseudobassiana (S.A.Rehner\&Humber) (Hypocreales: Cordycipitaceae), procedente de Cuevas del Valle (Ávila), sobre $M$. galloprovincialis. La capacidad de infección de este tipo de hongos entomopatógenos está limitada por su reducida capacidad de dispersión en las masas forestales (Shah and Pell, 2003). El uso forestal operativo de estos agentes se encuentra condicionado por la posibilidad de su aplicación práctica.

Las técnicas de autodiseminación, como "atrae\&infecta", constituyen una posibilidad para la aplicación de estos agentes. Pretenden la atracción de los propios insectos a estaciones donde son autoinfectados con el hongo entomopatógeno y autoliberados, de forma que antes de su muerte son capaces de dispersar el inóculo en su población. La disponibilidad de un cebo atractivo y de un agente biológico virulento ofrece una oportunidad de desarrollar esta técnica para el control de las poblaciones de M. galloprovincialis en áreas de especial interés.

\section{Objetivos}

El objetivo principal es desarrollar un sistema de autoinfección de M. galloprovincialis con B. pseudobassiana que pueda aplicarse operativamente para manejar la EMP en zonas demarcadas o de alto riesgo. Mediante los siguientes objetivos particulares:

- Desarrollar un sistema de autoliberación de $M$. galloprovincialis desde el bote colector extended de trampas multiembudos (Econex Multifunnel-12 ${ }^{\circledR}$ ) cebadas con Galloprotect-2D ${ }^{\circledR}$ (SEDQ S.L.).

- Desarrollar un sistema de autoinfección de M. galloprovincialis con B. pseudobassiana compatible con el dispositivo de autoliberación.

- Aumentar la selectividad del método frente a especies no objetivo capturadas.

- Determinar la dosis de B. pseudobassiana que permita la autoinfección del insecto y su transmisión utilizando los dispositivos de autoinfección. 


\section{Material y métodos}

\subsection{Sitios experimentales}

Dos experimentos de campo ( 1 y 2$)$ se llevaron a cabo en fustal natural de $P$. pinaster en resinación en los montes MUP 54 y MUP 224 (30N X=381.200, Y= 4.581.200) (Vallelado, Segovia). El Experimento 3 se realizó en el laboratorio de plagas del iuFOR-UVa.

\subsection{Dispositivo de autoliberación y autoinfección}

Se diseñó un sistema que permitiese la autoliberación de M. galloprovincialis desde el bote colector "extended" teflonado (diámetro $10 \mathrm{~cm}$, profundidad $23 \mathrm{~cm}$ ) de la trampa multiembudos (Econex-Multifunnel ${ }^{\circledast}$; Econex; Murcia) y, simultáneamente, se autoinfectase.

La modificación consistió en acoplar un tubo Falcón al que se le cortó la base y se perforó su tapa (65 $\mathrm{mm}$ de longitud y $27 \mathrm{~mm}$ de diámetro interno). La tapa se unió mediante cola termofusible a la pared exterior del bote colector, coincidiendo con un orificio de $27 \mathrm{~mm}$ de diámetro perforado previamente. La unión roscada de la tapa permite enroscar el trozo de tubo, abierto en sus dos extremos, quedando una vía de escape para los insectos desde el interior del bote colector.(Figura 2).

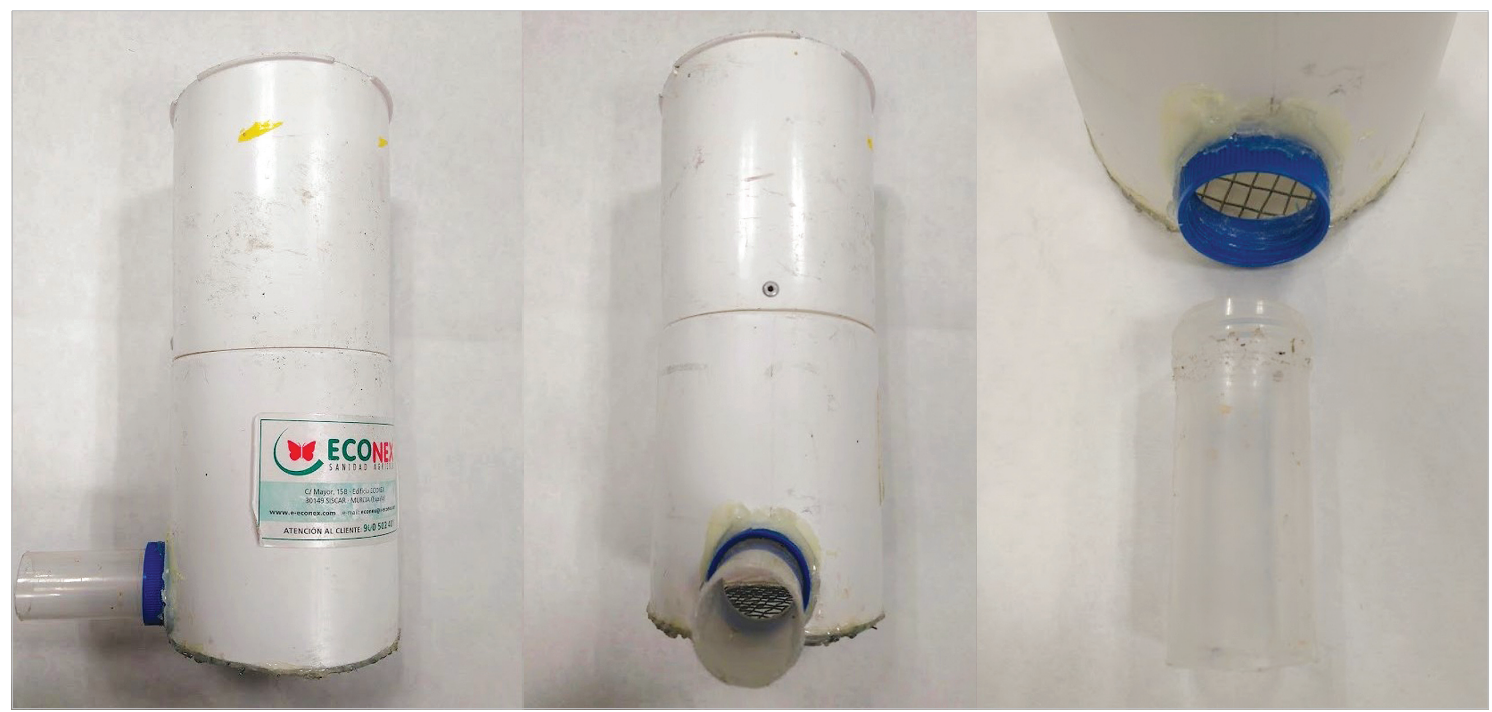

Figura 2. Prototipo original del dispositivo de autoliberación. Perfil (izquierda), alzado (centro) y detalle de la unión entre bote y dispositivo a través de la tapa perforada (derecha).

El dispositivo de autoliberación puede realizar la función de autoinfección recubriendo la pared interna con un fieltro (Okitsu et al., 2000; Shimazu and Sato, 2003), en el que se aplica una formulación de conidios en talco. El insecto al escapar por el dispositivo camina y roza contra el talco y se contamina con los conidios (Figura 3). 


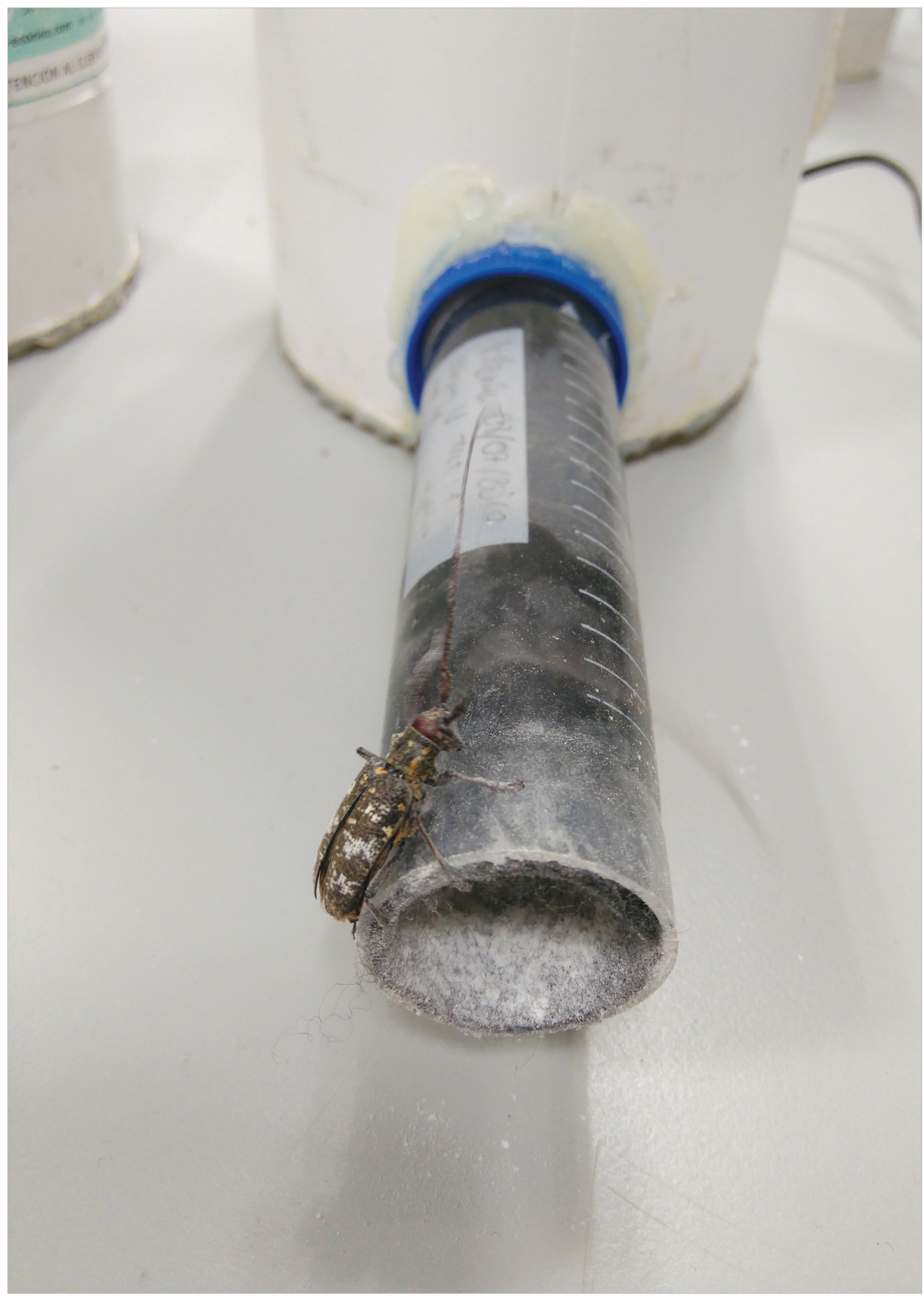

Figura 3. Funcionamiento del dispositivo de autoinfección. M. galloprovincialis que acaba de autoliberarse y autoinfectarse con talco, prueba realizada en laboratorio. 
Para determinar los individuos capturados autoliberados se acopló un bote accesorio (diámetro $10 \mathrm{~cm}$, altura $15 \mathrm{~cm}$ ) a la salida del tubo, de forma que los insectos autoliberados a través de éste eran recogidos en el bote accesorio (Figura 4).
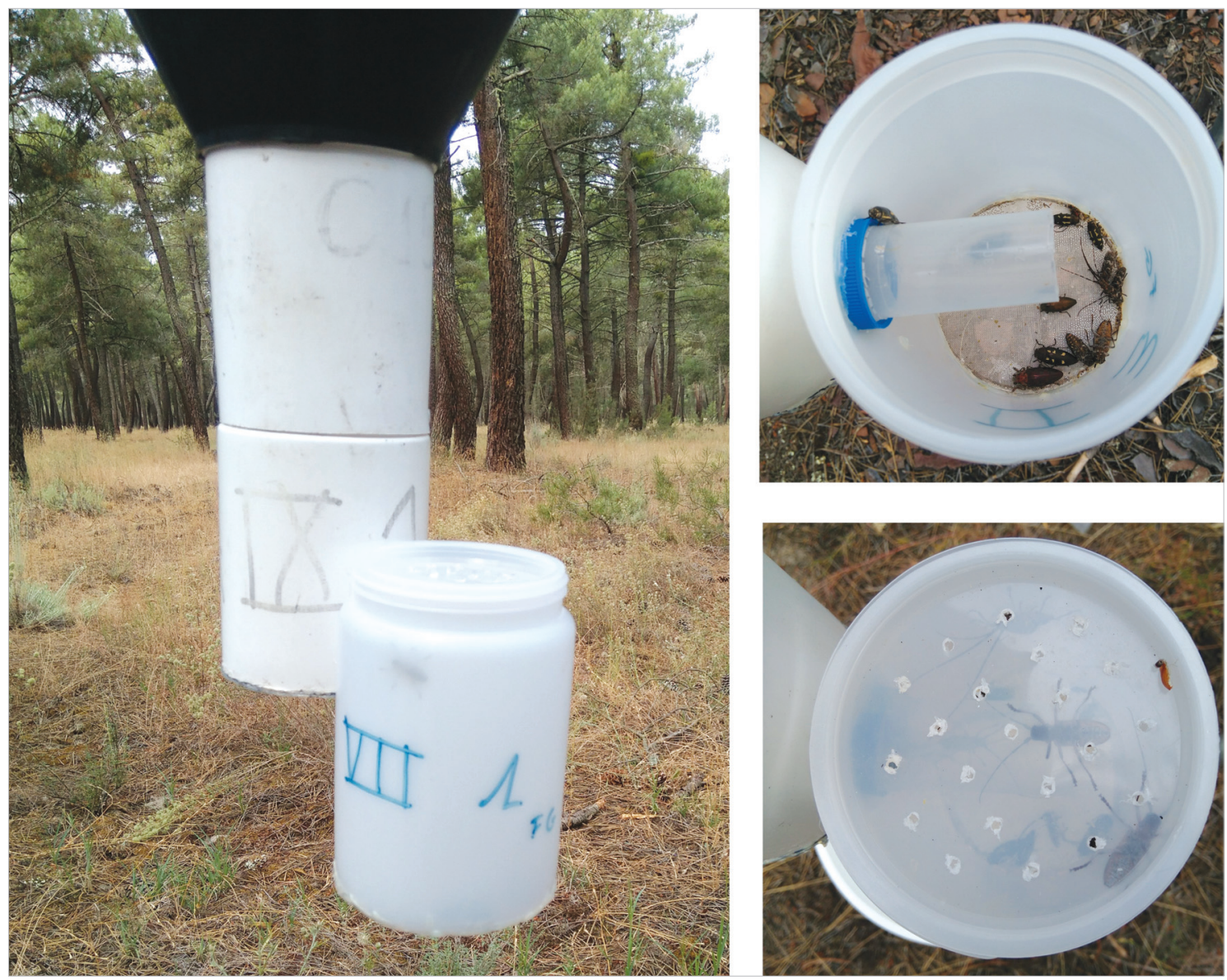

Figura 4. Bote auxiliar unido a prototipo del dispositivo de autoliberación (izquierda). Detalle de unión con el prototipo (la tapa azul se emplea de unión entre bote colector y bote auxiliar), detalle de dispositivo de autoliberación y detalle de malla tupida en fondo y capturas (derecha arriba). Detalle de tapa perforada en bote auxiliar para permitir la aireación (derecha abajo).

En los experimentos de campo se utilizaron trampas multiembudos provistas de bote colector "extended" teflonado (Econex Multifunnel ${ }^{\circledR} 12$; Murcia) suspendidas a 2 metros de altura y cebadas con Galloprotect-2D ${ }^{\circledR}$ (SEDQ SL., Barcelona).

\subsection{Experimentos}

Experimento 1. Efectividad del dispositivo de autoliberación y reducción del impacto sobre especies no objetivo.

Se evaluó la efectividad del dispositivo de autoliberación. Para evitar el potencial impacto sobre especies no objetivo la malla tupida del fondo del bote colector 
fue sustituida por una de mayor luz. Se testaron: Control (C), Malla Estrecha (ME): 4,8 mm, y Malla Ancha (MA): 6,0 mm (Figura 5).
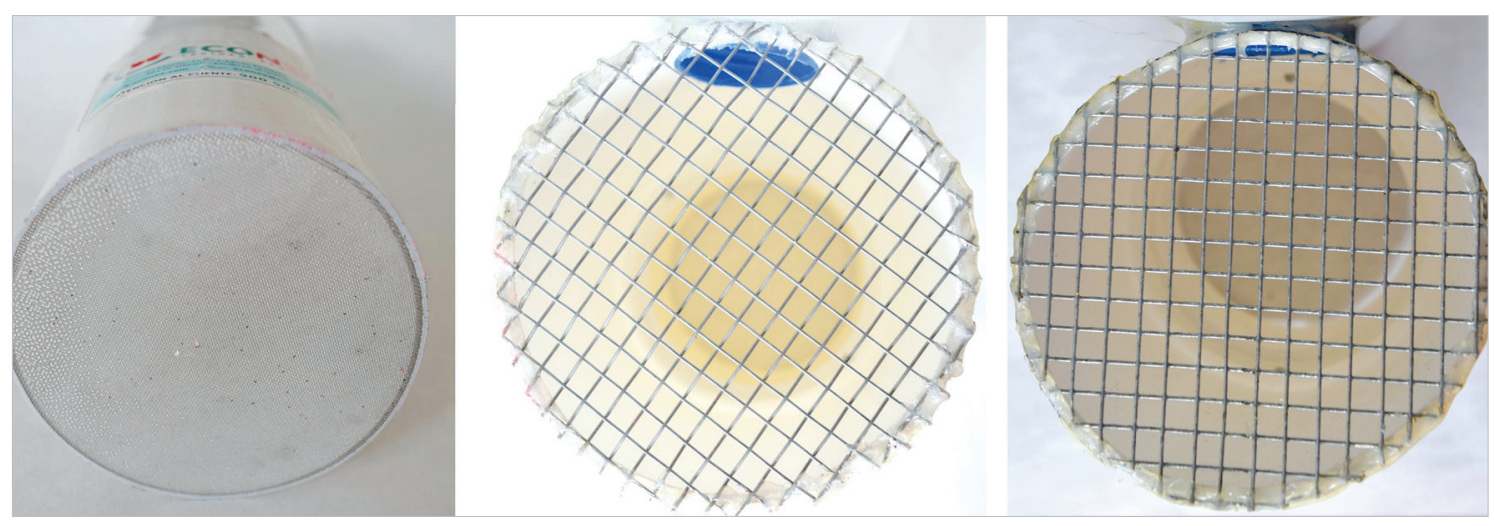

Figura 5. Detalle de las mallas que definen los tratamientos del experimento 1. Control (C): malla estándar del bote colector Econex (izquierda), Malla Estrecha (ME): malla de luz 4,8 mm (centro) y Malla Ancha (MA): malla de luz 6,0 mm (derecha).

El diseño experimental consistió en siete bloques completos al azar sin aleatorización. La separación entre trampas fue de $80 \mathrm{~m}$ y entre bloques $300 \mathrm{~m}$. El experimento se desarrolló durante julio de 2016 muestreado semanalmente.

Experimento 2. Aumento de la selectividad de la trampa frente a especies no objetivo.

Se pretendió aumentar la selectividad de especies no objetivo elevando la conexión del dispositivo de autoliberación $1,0 \mathrm{~cm}$ por encima del fondo del bote colector (Figura 6). Se intentó evitar que los individuos no objetivo que no podían escapar a través de la malla del fondo (MA) del bote colector lo hiciesen por el dispositivo de autoliberación. Se testaron Tubo Bajo (TB): dispositivo conectado a ras del fondo, con bote auxiliar, Tubo Alto (TA): dispositivo conectado $1 \mathrm{~cm}$ por encima del fondo, con bote auxiliar y Control (C): bote colector "Econex extended" teflonado.

El diseño experimental y muestreo fue similar al Experimento 1, entre el 25 de julio y el 10 de octubre de 2016.

Experimento 3. Autoinfección de Monochamus galloprovincialis con Beauveria pseudobassiana en laboratorio.

Se evaluó la efectividad de los dispositivos de autoinfección con una formulación de B. pseudobassiana EABpb en talco midiendo el tiempo de supervivencia de los individuos autoinfectados en laboratorio.

Se testaron dos dispositivos: dispositivo estrecho (EF), $27 \mathrm{~mm}$ diámetro con filtro fino $(1,5 \mathrm{~mm}$ espesor) y dispositivo ancho $(\mathrm{AG}), 36 \mathrm{~mm}$ diámetro con fieltro 


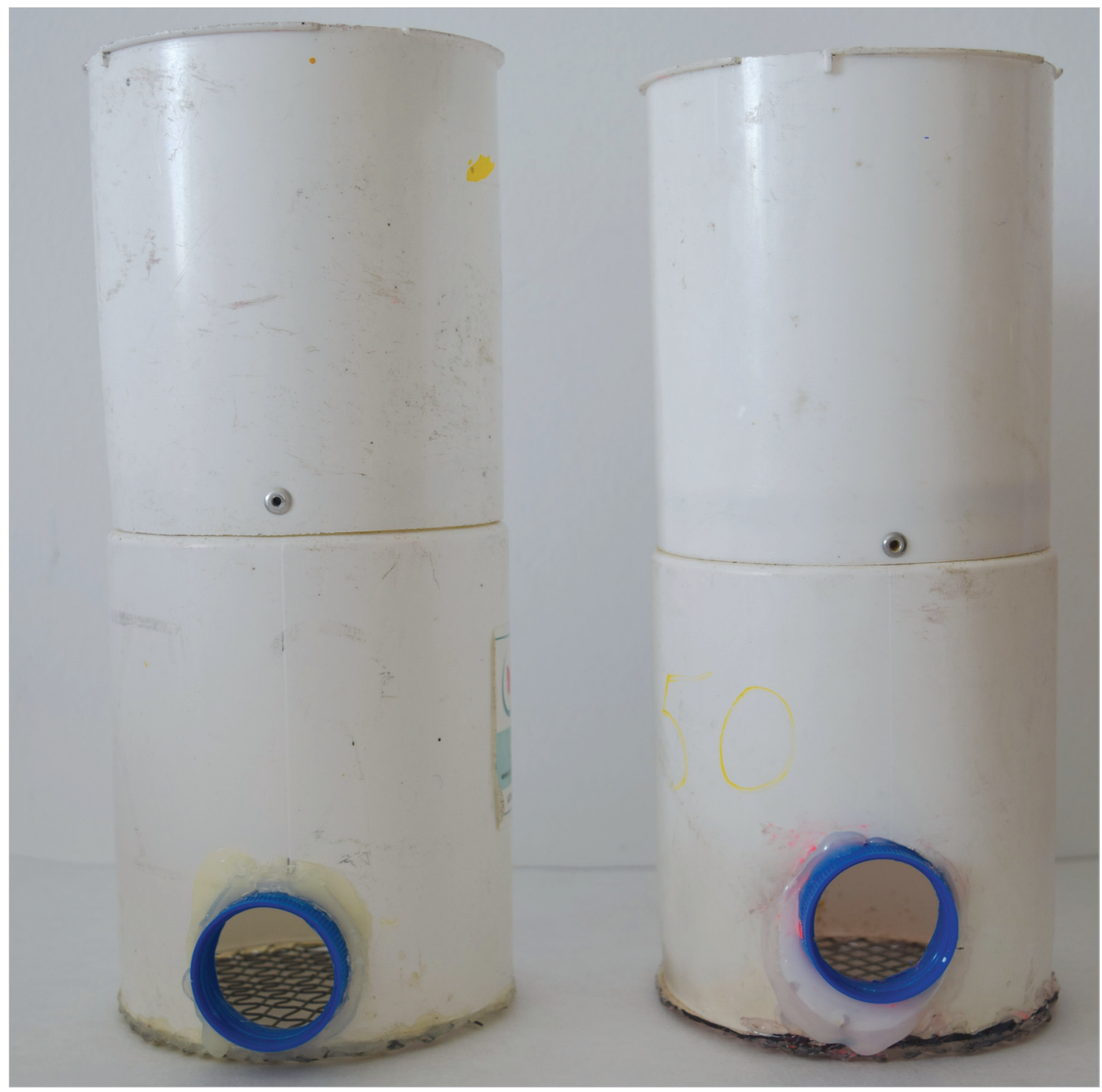

Figura 6. Diferentes alturas de conexión del dispositivo de autoliberación. Tubo bajo (TB): a ras del fondo (izquierda). Tubo Alto (TA): elevado 1,0 cm por encima del fondo (derecha).

grueso (3,0 mm espesor) y cuatro dosis de inóculo: control blanco (C), control talco (T), $1 \cdot 10^{9}$ (B9) y $1 \cdot 10^{10}$ (B10) conidios B.p. gramo ${ }^{-1}$ en talco. En total se testaron 8 tratamientos en un diseño factorial (2 dispositivos x 4 dosis): AGC, EFC, AGT, AGC, AGB9, EFB9, AGB10 y EFB10.

La longitud de los tubos se aumentó a $100 \mathrm{~mm}$ (Figura 7). El inóculo infectivo fue elaborado en la Universidad de Córdoba.

Los 20 insectos ensayados por tratamiento procedían de Vallelado (Segovia) (Experimento 2) y Tabuyo (León). Los tratamientos se aplicaron a través del dispositivo de autoinfección. Los insectos se mantuvieron individualmente en botes 1 litro con ramillas de pino. Se inspeccionaron diariamente 21 días, determinando si estaban vivos y vitales, vivos sin vitalidad o muertos. 

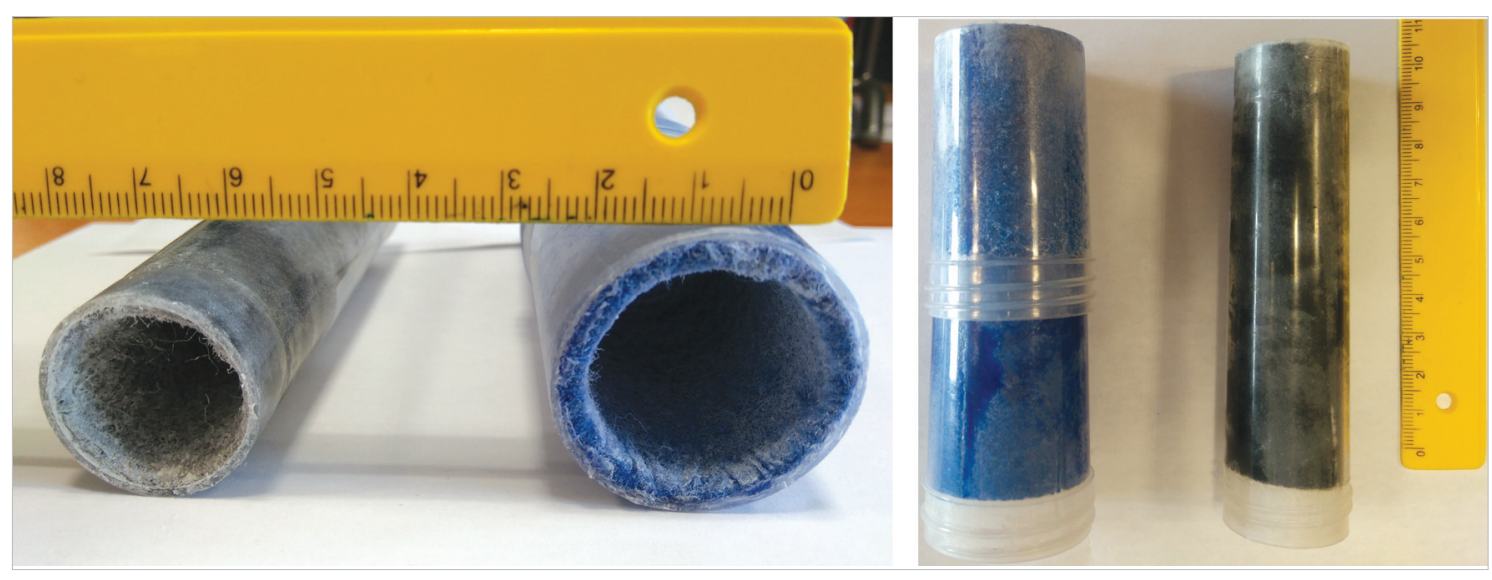

Figura 7. Detalle de dispositivos de autoinfección. Estrecho con fieltro fino (EF) (color negro) y ancho con fieltro grueso (AG) (color azul).

\subsection{Análisis estadístico}

Las variables respuesta medidas en los experimentos 1 y 2 fueron el número de individuos de las distintas especies capturadas en bote principal y auxiliar. Se analizaron mediante una ANOVA (GLM) para un diseño de bloques al azar y test de Tukey (nivel de confianza 95\%). Se utilizó el paquete R 3.3.3 (R Core Team, 2017).

En el experimento 3, se analizó el tiempo hasta que los insectos estaban vivos sin vitalidad y muertos. Se aplicó un GLM (distribución = binomial; link = logit) y test $\chi^{2}(\mathrm{P}<0,05)$ (JMP 8.0, 2008 SAS Institute Inc.). Se analizó mediante KaplanMeier para comparar el tiempo promedio de supervivencia (TPS) y el tiempo promedio de vitalidad (TPV) (Kaplan y Meier, 1958), con el software BIM ${ }^{\circledR}$ SPSS $^{\circledR}$ Statistics, v.24.

\section{Resultados}

Experimento 1. Efectividad del dispositivo de autoliberación y reducción del impacto sobre especies no objetivo.

Se comparan las capturas medias por trampa de M. galloprovincialis, sin diferencias significativas, indica que no se produjeron escapes a través de las mallas del fondo (Figura 8). Se observa que los M. galloprovincialis se autoliberaron a través del dispositivo (Figura 9). 


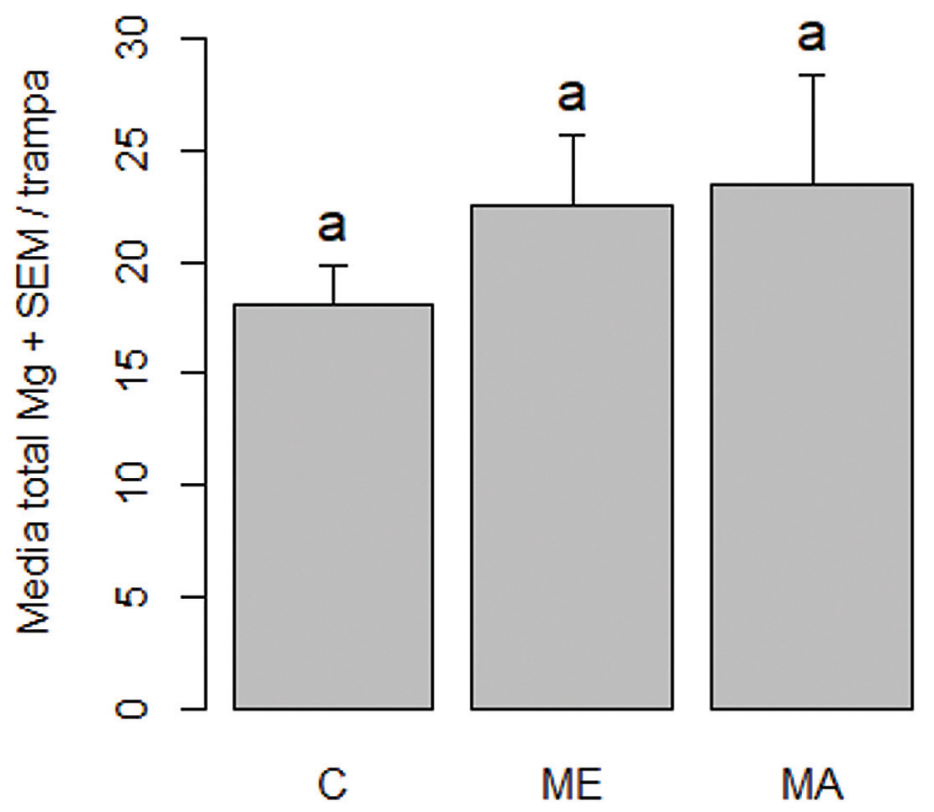

Tratamiento

Figura 8. Media M. galloprovincialis capturados por trampa en el Experimento 1. ME: Malla de luz 4,8 mm (malla estrecha), MA: Malla de luz 6,0 mm (malla ancha), C: Control (bote colector con malla estándar). Medias seguidas de la misma letra no son diferentes significativamente, $\mathrm{p}<0.05$ ).

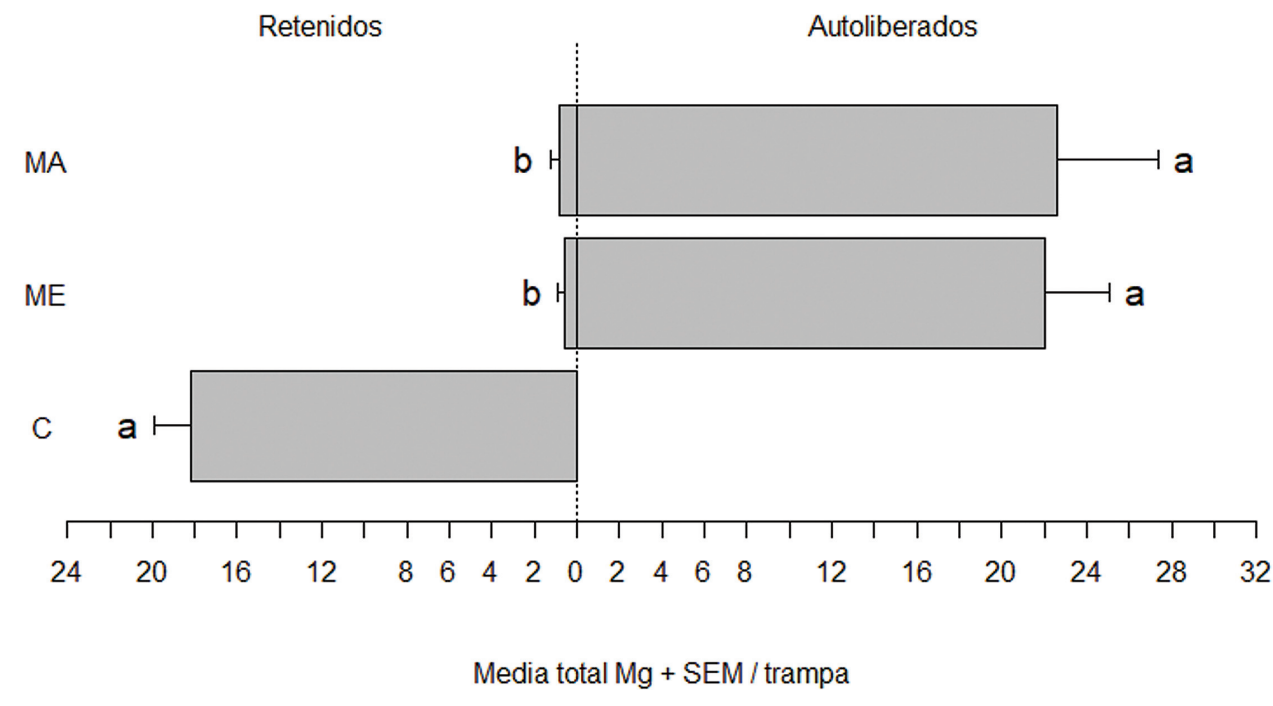

Figura 9. Media M. galloprovincialis capturados por trampa en el Experimento 1. Retenidos: individuos que permanecen en el bote colector. Autoliberados: individuos que escapan a través del dispositivo de autoliberación. ME: Malla de luz 4,8 mm (Malla Estecha), MA: Malla de luz 6,0 mm (Malla Ancha), C: Control (malla estándar). No existen individuos autoliberados en el tratamiento Control. Medias seguidas de la misma letra no son diferentes significativamente, $\mathrm{p}<0.05$ ). 
Se capturaron un total de 12 especies de insectos no objetivo, coleópteros saproxílicos (Tabla 1).

Tabla 1. Capturas de coleópteros saproxílicos no objetivo en los experimentos 1 y 2 .

D (depredador), X (xilófagos), XF (xilofungívoros) y SX (saproxilófagos). Anchura máx.: se mide en la parte más ancha de los élitros. (Bouget et al., 2005; Etxebeste et al., 2013).

\begin{tabular}{|c|c|c|c|c|c|}
\hline Familia & Género y especie Autor & Tipo $^{1}$ & $\begin{array}{l}\text { Anchura } \\
\text { máx. (mm) }\end{array}$ & $\begin{array}{c}\text { Captura } \\
\text { Exp.1 } \\
\text { (05 jul-25 jul })\end{array}$ & $\begin{array}{c}\text { Captura } \\
\text { Exp.2 } \\
(25 \text { jul-10 oct })\end{array}$ \\
\hline Trogossitidae & Temnochila caerulea Olivier & $\mathrm{D}$ & $3,0 / 5,0$ & 318 & 208 \\
\hline Cleridae & Thanasimus formicarius L. & $\mathrm{D}$ & $1,5 / 3,0$ & 164 & 16 \\
\hline \multirow{2}{*}{ Elateridae } & Lacon punctatus Herbst & SX & $3,0 / 5,5$ & 4 & 2 \\
\hline & Stenagostus rufus De Geer & SX & $4,0 / 5,5$ & 2 & 2 \\
\hline \multirow{5}{*}{ Cerambycidae } & Spondylus buprestoides $\mathrm{G}$. & SX & $4,0 / 8,0$ & 105 & 224 \\
\hline & Acanthocinus aedilis $\mathrm{L}$. & SX & $1,5 / 3,0$ & 3 & 4 \\
\hline & Arhopalus ferus Mulsant & $\mathrm{X}$ & $2,0 / 4,0$ & 0 & 12 \\
\hline & Hylotrupes bajulus L. & SX & $4,0 / 6,0$ & 7 & 1 \\
\hline & Xylotrechus arvicola Olivier & $\mathrm{X}$ & $3,0 / 4,0$ & 1 & 0 \\
\hline \multirow{3}{*}{ Buprestidae } & Buprestis novemmaculata L. & $\mathrm{X}$ & $5,0 / 9,0$ & 93 & 68 \\
\hline & Buprestis rustica $\mathrm{L}$. & $\mathrm{X}$ & $5,0 / 9,0$ & 24 & 41 \\
\hline & Chalcophora mariana $\mathrm{L}$. & $\mathrm{X}$ & $9,0 / 11,0$ & 64 & 42 \\
\hline
\end{tabular}

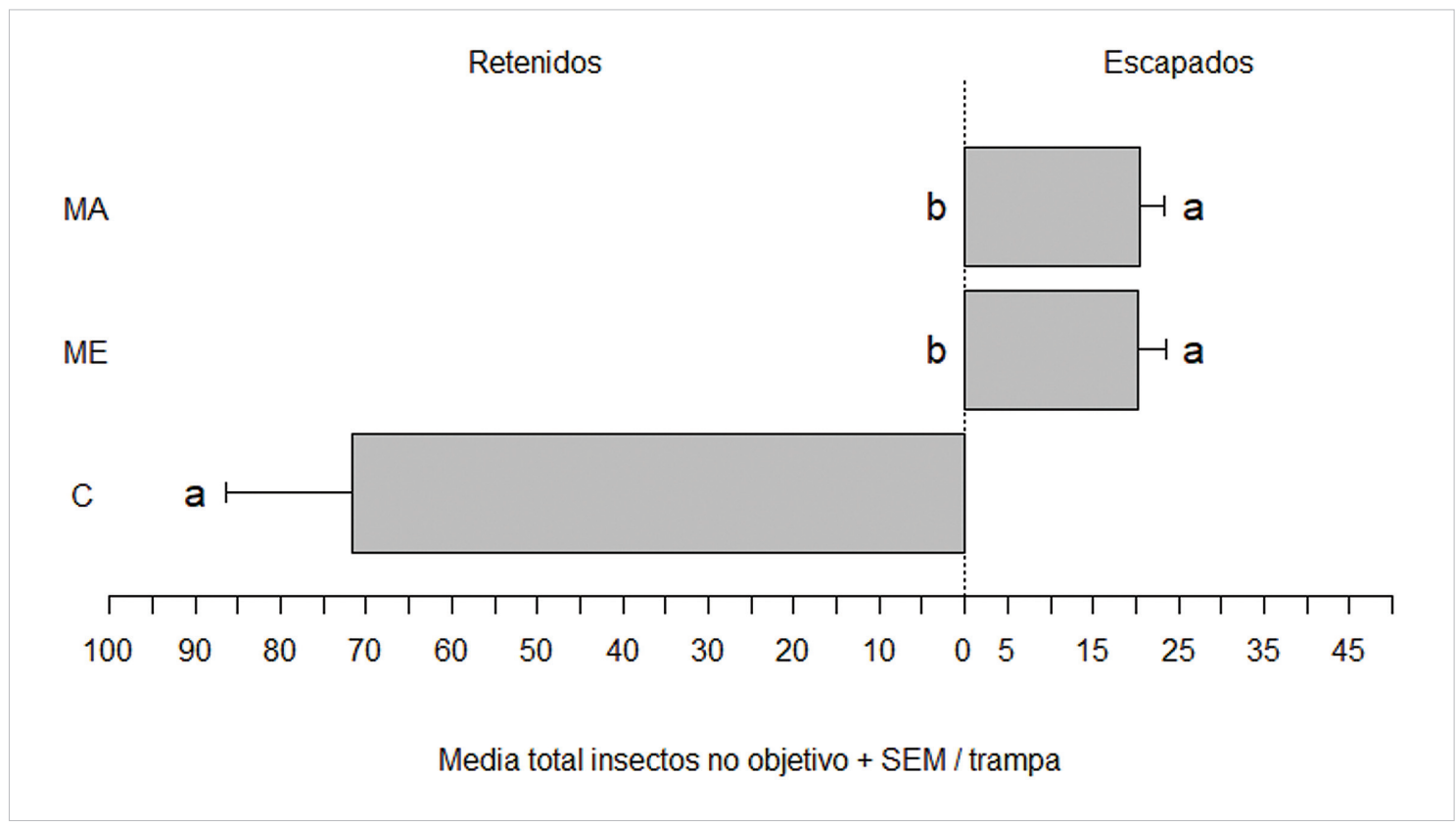

Figura 10. Media de individuos por trampa de todas las especies no objetivo capturadas en el tratamiento control, o que utilizaron el dispositivo de autoliberación para escapar en los tratamientos ME (Malla Estrecha) y MA (Malla Ancha) del experimento 1. Medias seguidas de la misma letra no son diferentes significativamente, $\mathrm{p}<0.05)$. 
Al comparar las capturas totales de insectos no objetivo, se determina que un $72 \%$ escaparon a través del fondo (ME y MA), sin diferencias significativas entre mallas (Figura 10), el 28\% restante escaparon por el dispositivo de autoliberación de M. galloprovincialis y habrían sido infectados con el hongo entomopatógeno.

\section{Experimento 2. Aumento de la selectividad de la trampa frente a especies} no objetivo.

En las Figuras 11 y 12 se recogen las capturas de M. galloprovincialis obtenidas en los tratamientos Control, TA y TB. No aparecen diferencias significativas (Figura 11), lo que indica ningún M. galloprovincialis escapó a través de la MA, todos se autoliberaron, sin diferencias entre el bote conectado al ras de fondo (TB) o $1 \mathrm{~cm}$ por encima (TA).

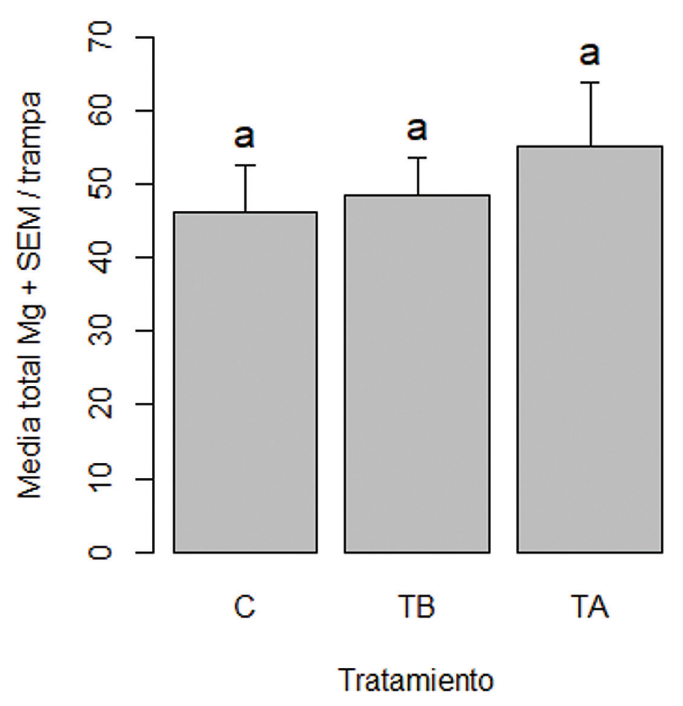

Figura 11. Media de M. galloprovincialis capturados por trampa en el Experimento 2. TA: Tubo Alto (conectado 1,0 cm por encima del fondo); TB: Tubo Bajo (conectado enrasado con el fondo; C: Control (bote colector estándar). Medias seguidas de la misma letra no son diferentes significativamente, $\mathrm{p}<0.05$ ) 


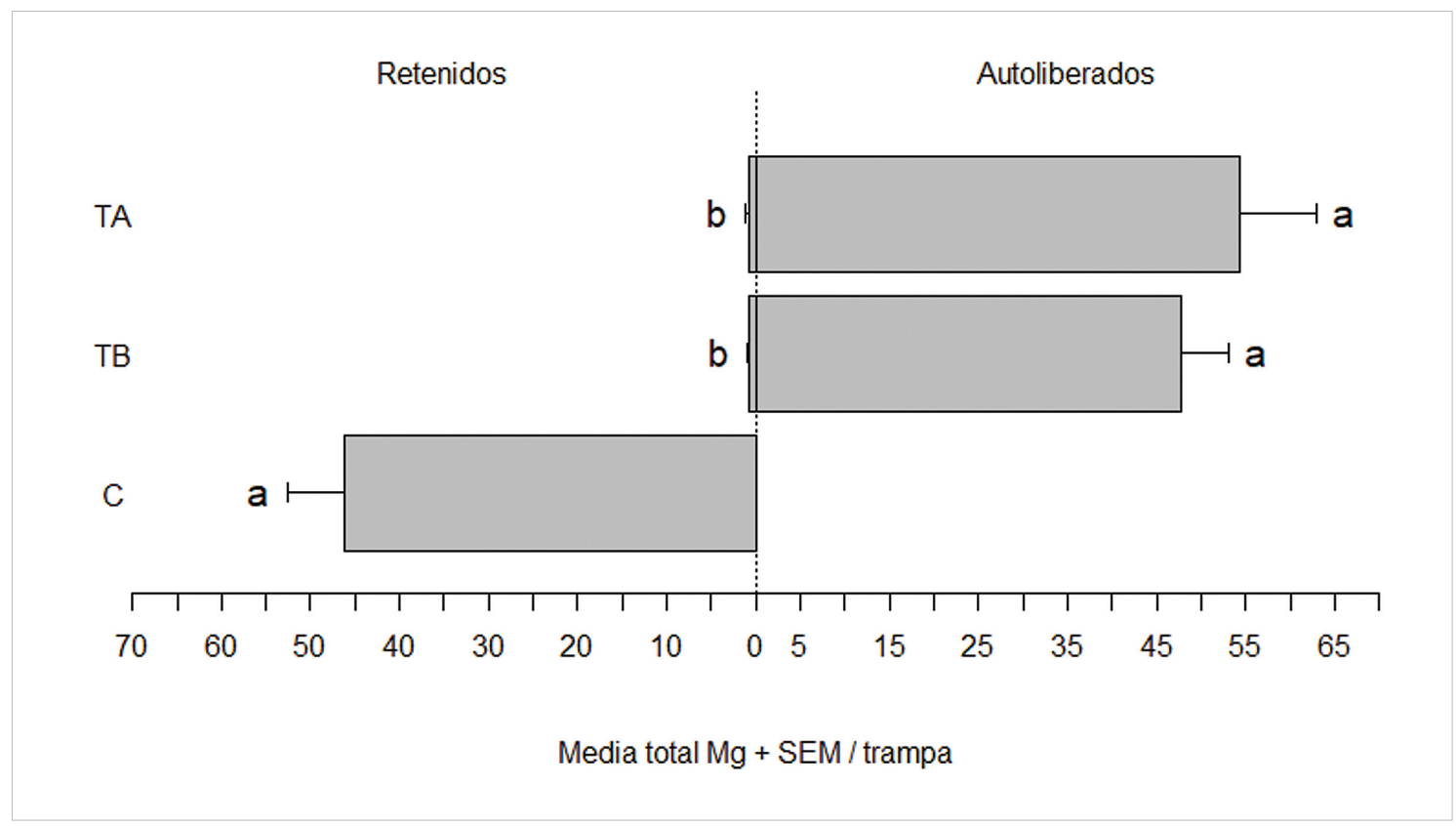

Figura 12. Media de M. galloprovincialis capturados por trampa en el Experimento 2. Retenidos: individuos que permanecen en el bote colector. Autoliberados: individuos que escapan a través del dispositivo de autoliberación. Tubo Alto (conectado 1,0 cm por encima del fondo); TB: Tubo Bajo (conectado enrasado con el fondo; C: Control (bote colector estándar). No existen individuos autoliberados en el tratamiento Control. Medias seguidas de la misma letra no son diferentes significativamente, $\mathrm{p}<0.05$ ).

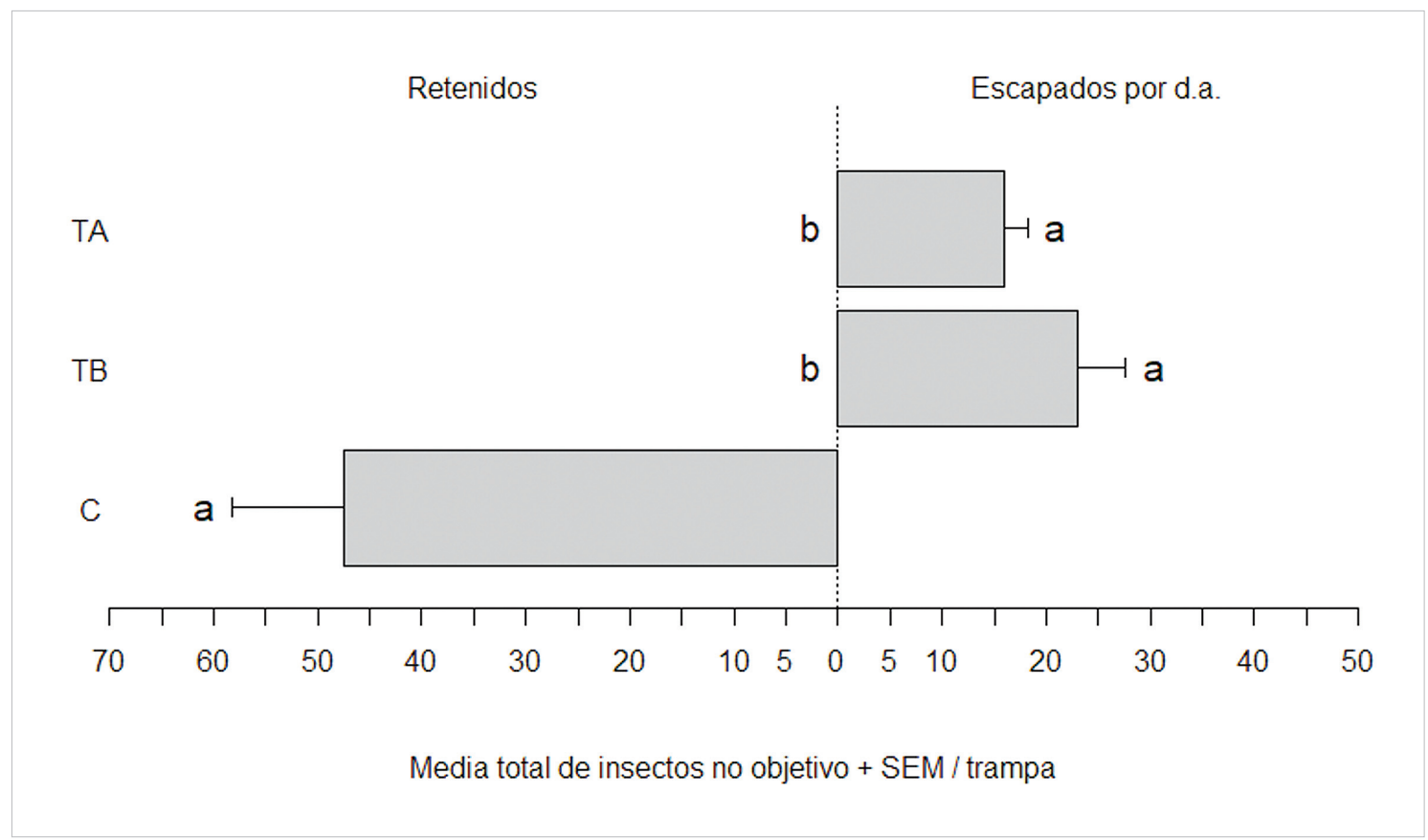

Figura 13. Media de individuos por trampa de todas las especies no objetivo capturadas en el tratamiento control, o que utilizaron el dispositivo de autoliberación TA: Tubo, TB: Tubo Bajo. Escapados por d.a.: Insectos no objetivo escapados a través del dispositivo de autoliberación de M. galloprovincialis. Tratamientos con la misma letra no son diferentes, $\mathrm{p}<0.05$ ). 
Se observó una ligera reducción no significativa en el número de insectos no objetivo que escaparon a través del dispositivo elevado (TA) comparado con el dispositivo bajo (Figura 13). Esto indica que la elevación del dispositivo de autoliberación para $M$. galloprovincialis podría suponer una mejora en la selectividad del sistema la trampa evitando que otras especies escapasen a través de él y escapasen por el fondo.

Si se analizan los resultados por especies no objetivo (Tabla 2) es destacable la reducción general en las capturas de los depredadores T. formicarius y T. caerulea.

Tabla 2. Media de individuos por trampa de cada una de las especies no objetivo capturadas en el tratamiento control, o que utilizaron el dispositivo de autoliberación para escapar en los tratamientos TA: Tubo Alto (1,0 cm por encima del fondo) o TB: Tubo Bajo (enrasado con el fondo del experimento. 2. Medias para cada especie seguidas de la misma letra no son diferentes significativamente, $\mathrm{p}<0.05$ ).

\begin{tabular}{lccc}
\hline \multicolumn{1}{c}{ Género y especie Autor } & Control & $\begin{array}{c}\text { Escapados a través } \\
\text { de dispositivo (TB) }\end{array}$ & $\begin{array}{c}\text { Escapados a través } \\
\text { de dispositivo (TA) }\end{array}$ \\
\hline Buprestis novemmaculata (L.) & $0,30 \pm 0,37 \mathrm{a}$ & $0,34 \pm 0,30 \mathrm{a}$ & $0,25 \pm 0,25 \mathrm{a}$ \\
\hline Buprestis rustica (L.) & $0,14 \pm 0,16 \mathrm{a}$ & $0,23 \pm 0,26 \mathrm{a}$ & $0,14 \pm 0,13 \mathrm{a}$ \\
\hline Chalcophora mariana (L.) & $0,21 \pm 0,19 \mathrm{a}$ & $0,21 \pm 0,21 \mathrm{a}$ & $0,13 \pm 0,13 \mathrm{a}$ \\
\hline Spondylus buprestoides (Gressitt) & $0,82 \pm 0,38 \mathrm{a}$ & $1,26 \pm 0,54 \mathrm{a}$ & $0,83 \pm 0,37 \mathrm{a}$ \\
\hline Thanasimus formicarius (L.) & $0,16 \pm 0,20 \mathrm{a}$ & $0,00 \pm 0,00 \mathrm{~b}$ & $0,05 \pm 0,08 \mathrm{ab}$ \\
\hline Temnochila caerulea (Olivier) & $2,60 \pm 2,25 \mathrm{a}$ & $0,05 \pm 0,08 \mathrm{~b}$ & $0,05 \pm 0,08 \mathrm{~b}$ \\
\hline
\end{tabular}

Experimento 3. Autoinfección de Monochamus galloprovincialis con Beauveria pseudobassiana en laboratorio.

Los individuos autoinfectados ensayados recogieron dosis de inóculo significativamente diferentes (Tabla 3). Todos los tratamientos con B. pseudobassiana causaron la mortalidad total de los insectos tratados, mientras que la mortalidad de los

Tabla 3. Dosis de inóculo recogidas por los individuos de M. galloprovincialis al recorrer el dispositivo de autoinfección.

Ver Material y Métodos para una descripción de los tratamientos.

Medias seguidas de la misma letra no son diferentes significativamente, $\mathrm{p}<0.05$ ).

\begin{tabular}{lcc}
\hline Tratamiento & $\begin{array}{c}\text { Dosis media } \\
(\mathrm{mg} / \text { insecto })\end{array}$ & $\begin{array}{c}\text { Dosis/peso } \\
(\%)\end{array}$ \\
\hline AGT & $1,58 \mathrm{a}$ & 0,59 \\
AGB9 & $1,85 \mathrm{a}$ & 0,62 \\
AGB10 & $1,61 \mathrm{a}$ & 0,55 \\
EFT & $2,02 \mathrm{~b}$ & 0,88 \\
EFB9 & $2,69 \mathrm{~b}$ & 0,93 \\
EFB10 & $2,70 \mathrm{~b}$ & 0,81 \\
\hline
\end{tabular}


controles fue sólo la mitad y su TPS significativamente mayor (Tabla 4). Se encontraron diferencias significativas en el TPS de los individuos entre tratamientos. Los insectos infectados por la dosis B9 a través del dispositivo EF sobrevivieron menos tiempo que los infectados por esta dosis en el dispositivo AG.

Tabla 4. Mortalidad y Tiempo Promedio de Supervivencia (TPS) para cada tratamiento de autoinfección con B. pseudobassiana.

Medias con la misma letra no son significativamente diferentes entre sí, $(\mathrm{P}<0,05)$.

\begin{tabular}{|c|c|c|c|c|}
\hline \multirow{3}{*}{ Tratamiento } & \multirow{3}{*}{$\begin{array}{c}\text { Mortalidad } \\
(\%)\end{array}$} & \multicolumn{3}{|c|}{ Análisis de supervivencia de Kaplan-Meier } \\
\hline & & \multirow{2}{*}{$\begin{array}{c}\text { TPS } \\
(\text { media } \pm \text { SE) }\end{array}$} & \multicolumn{2}{|c|}{ Intervalo de confianza $(95 \%)$} \\
\hline & & & Inferior & Superior \\
\hline AGC & $50 \quad \mathrm{a}$ & $15,80 \pm 1,719 \quad \mathrm{a}$ & 12,432 & 19,168 \\
\hline AGT & 60 & $16,55 \pm 1,301 \quad \mathrm{a}$ & 13,999 & 19,101 \\
\hline AGB9 & $100 \mathrm{~b}$ & $12,15 \pm 0,871 \quad b$ & 10,442 & 13,858 \\
\hline AGB10 & $100 \mathrm{~b}$ & $6,65 \pm 0,412 \quad c$ & 5,842 & 7,458 \\
\hline EFC & $50 \quad \mathrm{a}$ & $16,35 \pm 1,469 \quad \mathrm{a}$ & 13,471 & 19,229 \\
\hline EFT & 45 & $17,50 \pm 1,048 \quad \mathrm{a}$ & 15,447 & 19,553 \\
\hline EFB9 & 100 & $8,85 \pm 0,689 \quad d$ & 7,499 & 10,201 \\
\hline EFB10 & 100 & $5,75 \pm 0,347 \quad c$ & 5,070 & 6,430 \\
\hline
\end{tabular}

En la Figura 14 se recogen las funciones de supervivencia para cada uno de los tratamientos del ensayo.

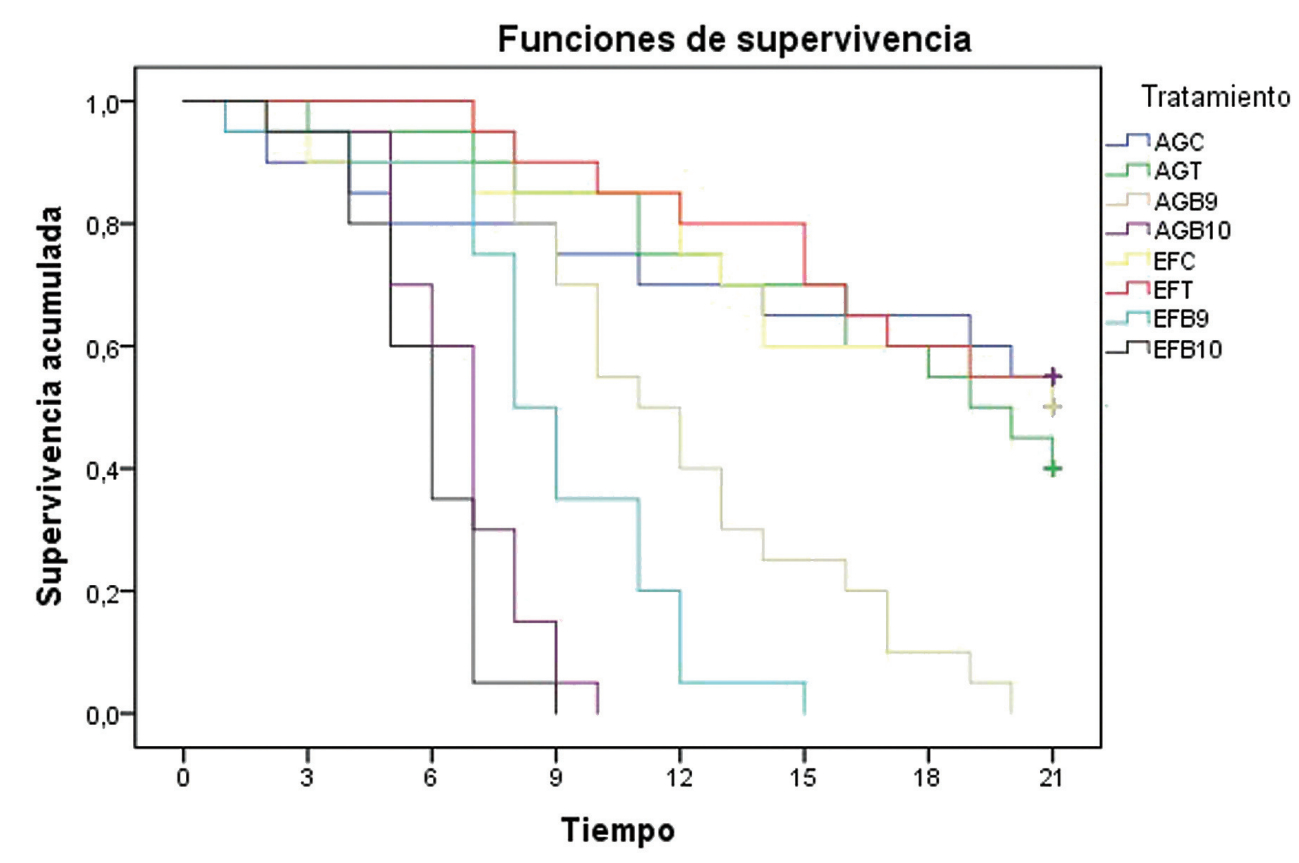

Figura 14. Funciones de supervivencia para cada tratamiento. Ver Material y métodos para una descripción de los tratamientos. 
Se analizó el tiempo en el que los individuos tratados se encontraron suficientemente vitales como para reproducirse (Tabla 5), obteniendo las mismas diferencias que el análisis de supervivencia, aunque el TPV fue inferior. Los tratamientos con menor dosis obtuvieron TPV de 4,9 (EF) o 6,8 (AG) días, acorde con los objetivos.

Tabla 5. Tiempo medio en el que los individuos tratados se muestran vitales (realizan movimientos con todos los artejos).

${ }^{1}$ TPV: Tiempo Promedio de Vitalidad limitado a 21 días. Los datos en misma columna con misma letra no son significativamente diferentes $(\alpha=0,05)$.

\begin{tabular}{|c|c|c|c|c|}
\hline \multirow{3}{*}{ Tratamiento } & \multicolumn{4}{|c|}{ Análisis de vitalidad (modificado de TPS Kaplan-Meier) } \\
\hline & \multirow{2}{*}{\multicolumn{2}{|c|}{$\begin{array}{c}\text { TPV } \\
(\text { media } \pm \text { SE) }\end{array}$}} & \multicolumn{2}{|c|}{ Intervalo de confianza $(95 \%)$} \\
\hline & & & Inferior & Superior \\
\hline AGC & $15,05 \pm 1,776$ & $\mathrm{a}$ & 11,569 & 18,531 \\
\hline AGT & $15,30 \pm 1,338$ & a & 12,677 & 17,923 \\
\hline AGB9 & $6,80 \pm 0,462$ & $\mathrm{~b}$ & 5,894 & 7,706 \\
\hline AGB10 & $3,15 \pm 0,233$ & $\mathrm{c}$ & 2,694 & 3,606 \\
\hline $\mathrm{EFC}$ & $15,50 \pm 1,538$ & $\mathrm{a}$ & 12,136 & 18,164 \\
\hline EFT & $17,05 \pm 1,233$ & a & 14,634 & 19,466 \\
\hline EFB9 & $4,85 \pm 0,350$ & d & 4,164 & 5,536 \\
\hline EFB10 & $2,70 \pm 0,231$ & $\mathrm{c}$ & 2,248 & 3,152 \\
\hline
\end{tabular}

En la Figura 15 se relaciona la dosis de conidios por unidad de peso recibida por los individuos con el tiempo que permanecieron vitales, con capacidad de rea-

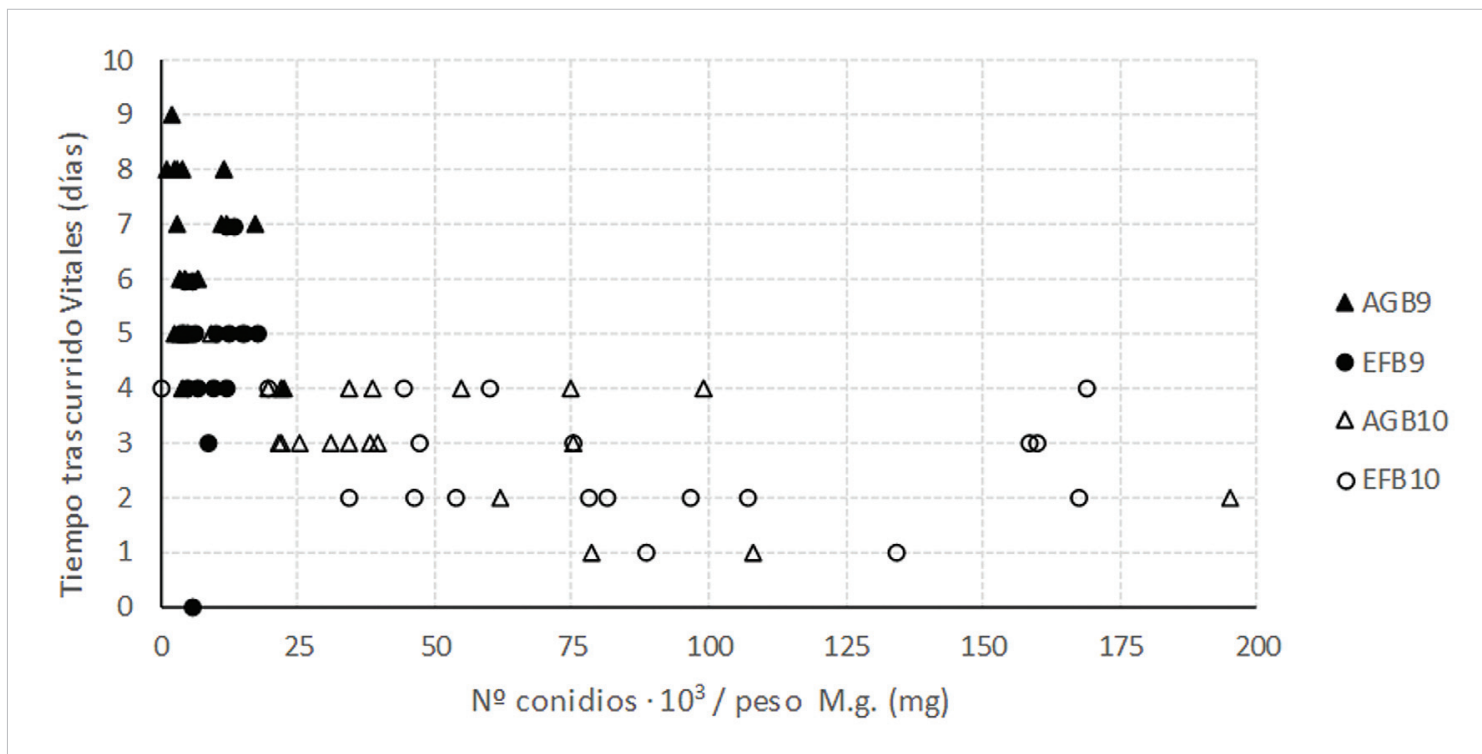

Figura 15. Relación entre tiempo promedio de vitalidad de M. galloprovincialis y dosis de conidios recibida por unidad de peso (mg). 
lizar un comportamiento normal, que permitiese la autodiseminación del entomopatógeno. Se observa una disminución exponencial del número de días vitales al aumentar la dosis recibida, hasta valores de $50 \cdot 10^{3}$ conidios $\cdot \mathrm{mg}^{-1}$ de peso. El aumento de esta dosis no supuso reducción apreciable del TPV.

\section{Discusión}

Los experimentos 1 y 2 demostraron el correcto funcionamiento del sistema de autoliberación para M. galloprovincialis. Los imagos fueron capaces de encontrar el acceso al dispositivo de autoliberación y usarlo.

Ambos experimentos evaluaron la efectividad del escape de especies no objetivo a través de mallas de fondo del bote colector que les permitiese su paso, pero que retuviesen a los $M$. galloprovincialis capturados. Ambas mallas ensayadas $(4,8$ $\mathrm{mm}$ y $6,0 \mathrm{~mm}$ ) resultaron efectivas para el escape de insectos no objetivo, cuyas capturas se vieron reducidas al $25 \%$, principalmente por el escape de T. caerulea y T. formicarius. (Ross and Daterman, 1998; Martín et al., 2013) han demostrado también la efectividad de mallas en la exclusión de depredadores de escolítidos en trampas multiembudos. Otras especies saproxílicas de tamaños mayores no pudieron salir a través de las mallas y escaparon del bote a través del dispositivo de autoliberación, lo que no resulta deseable. No aparecieron diferencias entre mallas, por lo que $6,0 \mathrm{~mm}$ sería aconsejable. El intento de dificultar el escape de insectos no objetivo por el dispositivo, mediante la elevación de $1 \mathrm{~cm}$ sobre el fondo, sin afectar a la autoliberación de la especie objetivo, no obtuvo diferencias significativas entre ambos tratamientos (TB y TA), aunque el número de capturas no objetivo fue ligeramente inferior en el TA.

En el Experimento 3 se determinó que un dispositivo de menor diámetro interno aporta una cantidad de inoculo significativamente mayor. Al atravesar el dispositivo EF, el individuo contacta en mayor medida en zona ventral, extremidades y élitros con la superficie inoculada. La cepa EABps de B. pseudobassiana confirmó su virulencia sobre M. galloprovincialis (Alvarez-Baz et al., 2015). Ambas dosis aplicadas con cualquier dispositivo redujeron significativamente el TPS de los individuos tratados, no obstante, los adultos ensayados se encontraban próximos al final de su vida, esto explica la mortalidad ocurrida en los controles. El tratamiento EFB9 obtuvo un TPV de 4,9 días y cantidad de inóculo transmisible de 2,7 mg/ insecto que le convierten en el candidato idóneo para la continuación de los estudios.

\section{Conclusiones}

Este trabajo muestra avances en el desarrollado un sistema efectivo para la autodiseminación del hongo entomopatógeno B. pseudobassiana sobre las poblaciones de M. galloprovincialis. 
- El sistema de autoliberación de M. galloprovincialis a través de un tubo enroscado externamente al bote colector de las trampas multiembudos ha resultado funcional. Este dispositivo es sencillo, barato, fácilmente recambiable y adaptable al bote colector de cualquier tipo de trampa.

- El escape de los insectos no objetivo capturados a través de mallas de $6,0 \mathrm{~mm}$ en el fondo del bote colector ha resultado exitoso para las especies depredadoras T. caerulea y T. formicarius. Debe mejorarse para otras especies de xilófagos de tamaño mayor.

- El dispositivo de autoliberación se ha transformado en un sistema efectivo de autoinfección mediante el recubrimiento interior del tubo con fieltro, donde inocular la formulación de conidios en talco.

- El dispositivo de autoinfección formado por un tubo de $27 \mathrm{~mm}$ de diámetro interior y $100 \mathrm{~mm}$ de longitud, con fieltro de $1,5 \mathrm{~mm}$ inoculado con una formulación en talco de $B$. pseudobassiana $1 \cdot 10^{9}$ conidios $\cdot \mathrm{g}^{-1}$, permite la adecuada supervivencia con vitalidad de los individuos de forma que podrían realizar una transmisión de inóculo en sus poblaciones.

Se deben continuar los estudios para determinar las condiciones de la transmisión horizontal y vertical y mejorar la selectividad del sistema.

\section{Agradecimientos}

Agradecer al laboratorio de Sanidad Vegetal perteneciente al Instituto universitario de Investigación en Gestión Forestal Sostenible (iuFOR) y Departamento de Producción Vegetal Sostenible de la Universidad de Valladolid todos los medios facilitados para llevar a cabo este trabajo, a Ana Ponce y Laura Ponce por su ayuda en los trabajos de campo y en el mantenimiento de los ejemplares de Monochamus galloprovincialis.

Mi gratitud a María Fernández Bravo y Enrique Quesada de la Universidad de Córdoba por la preparación del inoculo infectivo y auxilio con los análisis de mortalidad y supervivencia; al Servicio Territorial de Medio Ambiente de Segovia por las autorizaciones para el desarrollo de los ensayos de campo; al Centro de Sanidad Forestal de Calabazanos (Palencia) y a Gonzalo Álvarez por el apoyo logístico y colaboración en los análisis.

Este trabajo ha sido financiado el Ministerio de Economía, Industria y Competitividad a través del proyecto RTA2014-00042-C02-02. Durante el tiempo de realización del trabajo he estado financiado por el Plan de Contratación de personal para la promoción de empleo joven e implantación de la Garantía Juvenil I+D+i, del Ministerio de Economía, Industria y Competitividad. 


\section{Bibliografía}

Alvarez-Baz, G., Fernandez-Bravo, M., Pajares, J., Quesada-Moraga, E., 2015. Potential of native Beauveria pseudobassiana strain for biological control of Pine Wood Nematode vector Monochamus galloprovincialis. J. of invertebrate pathology, vol. 132, pp. 48-56. https://doi.org/10.1016/j.jip.2015.08.006

Alvarez, G., Etxebeste, I., Gallego, D., David, G., Bonifacio, L., Jactel, H., Sousa, E., Pajares, J.A., 2015. Optimization of traps for live trapping of Pine Wood Nematode vector Monochamus galloprovincialis. Journal of Applied Entomology, vol. 139, no. 8, pp. 618-626. https://doi.org/10.1111/jen.12186

Alvarez, G., Gallego, D., Hall, D.R., Jactel, H., Pajares, J.A., 2016. Combining pheromone and kairomones for effective trapping of the pine sawyer beetle Monochamus galloprovincialis. Journal of Applied Entomology, vol. 140, no. 1-2, pp. 58-71. https:// doi.org/ 10.1111/jen.12297

Bouget, C., Brustel, H., Nageleisen, L.M., 2005. Nomenclature des groupes écologiques d'insectes liés au bois: Synthèse et mise au point sémantique. Comptes Rendus - Biologies, vol. 328, no. 10-11, pp. 936-948. https://doi.org/10.1016/j.crvi.2005.08.003

EPPO, 2016. EPPO A1 and A2 Lists of pest recommended for regulation as quarentine pests. Vol. 2, no. September.

Etxebeste, I., Lencina, J.L., Pajares, J., 2013. Saproxylic community, guild and species responses to varying pheromone components of a pine bark beetle. Bulletin of entomological research, vol. 103, no. 5, pp. 497-510. https://doi.org/10.1017/S00074853120 00879

Fukuda, L., Fukuda, K., Hogetsu, T., Suzuki, K., 1992. Cavitation and Cytological Changes in Xylem of Pine Seedlings Inoculated with Virulent and Avirulent Isolates of Bursaphelenchus xylophilus and B. mucronatus. J. Jpn. For. Soc, vol. 74, no. 4, pp. 289-299.

Hara, N., Takeuchi, Y., Futai, K., 2006. Cytological changes in ray parenchyma cells of seedlings of three pine species infected with the pine wilt disease. Japanese Journal of Nematology, vol. 36, no. 1, pp. 23-32. https://doi.org/10.3725/jjn.36.23

Ibeas, F., Gallego, D., Diez, J.J., Pajares, J.A., 2007. An operative kairomonal lure for managing pine sawyer beetle Monochamus galloprovincialis (Coleoptera: Cerymbycidae). Journal of Applied Entomology, vol. 131, no. 1, pp. 13-20. https://doi.org/10.1111/ j.1439-0418.2006.01087.x

Kaplan, E.L., Meier, P., 1958. Nonparametric estimation from incomplete observations. Journal of the American Statistical Association, vol. 53, no. 282, pp. 457-481. https:// doi.org/10.1080/01621459.1958.10501452

Maehara, N., He, X., Shimazu, M., 2007. Maturation feeding and transmission of Bursaphelenchus xylophilus (Nematoda: Parasitaphelenchidae) by Monochamus alternatus (Coleoptera: Cerambycidae) inoculated with Beauveria bassiana (Deuteromycotina: Hyphomycetes). J Econ Entomol, vol. 100, no. 1, pp. 49-53. https://doi.org/10.1093/ jee/100.1.49

Mamiya, Y., 1983. Pathology of the Pine Wilt Disease caused by Bursaphelenchus xylophilus. Annual review of phytopathology, vol. 21, pp. 201-20. https://doi.org/10.1146/ annurev.py.21.090183.001221

Martín, A., Etxebeste, I., Pérez, G., Álvarez, G., Sánchez, E., Pajares, J., 2013. Modified 
pheromone traps help reduce bycatch of bark-beetle natural enemies. Agricultural and Forest Entomology, vol. 15, no. 1, pp. 86-97. https://doi.org/10.1111/j.14619563.2012.00594.x

Mas I Gisbert, H., 2015. Fenología y capacidad de dispersión de Monochamus galloprovincialis (Olivier 1795) en la península ibérica. Tesis Doctoral, ETSI Montes, Universidad Politécnica.

Mota, M., Braasch, H., Bravo, M.A., Penas, A.C., Burgermeister, W., Metge, K., Sousa, E., 1999. First report of Bursaphelenchus xylophilus in Portugal and in Europe. Nematology, vol. 1, no. December 2013, pp. 727-734. https://doi.org/10.1163/1568 54199508757

Naves, P.M., Sousa, E., Rodrigues, J.M., 2008. Biology of Monochamus galloprovincialis (Coleoptera, Cerambycidae) in the Pine Wilt Disease Affected Zone, Southern Portugal. Silva Lusitana, vol. 16, no. 2, pp. 133-148.

Pajares, J.A., Alvarez, G., Hall, D.R., Douglas, P., Centeno, F., Ibarra, N., Schroeder, M., Teale, S.A., Wang, Z., Yan, S., Millar, J.G., Hanks, L.M., 2013. 2-(Undecyloxy)-ethanol is a major component of the male-produced aggregation pheromone of Monochamus sutor. Entomologia Experimentalis et Applicata, vol. 149, no. 2, pp. 118-127. https:// doi.org/10.1111/eea.12113

Pajares, J.A., Alvarez, G., Ibeas, F., Gallego, D., Hall, D.R., Farman, D.I., 2010. Identification and field activity of a male-produced aggregation pheromone in the Pine Sawyer Beetle, Monochamus galloprovincialis. Journal of chemical ecology, vol. 36, no. 6, pp. 570-583. https://doi.org/10.1007/s10886-010-9791-5

Pajares, J.A., Ibeas, F., Gallego, D., 2004. Attractive responses by Monochamus galloprovincialis (Col.: Cerambycidae) to host and bark beetle semiochemicals. Journal of Applied Entomology, vol. 128, pp. 633-638. https://doi.org/10.1111/j.1439-0418.2004. 00899.x

R Core Team, 2017. R: A language and environment for statistical computing. R Foundation for Statistical Computing, Vienna, Austria. URL: http://www.R-project.org/

Ross, D.W., Daterman, D.E., 1998. Pheromone-baited traps Dendroctonus pseudotsugae (Col.: Scolytidae): Influenced of selected release rates and traps designs. Jour. Econ. Entom., vol. 91, pp. 500-506. https://doi.org/10.1093/jee/91.2.500

Shah, P.A., Pell, J.K., 2003. Entomopathogenic fungi as biological control agents. Applied Microbiology and Biotechnology, vol. 61, no. 5-6, pp. 413-423. https://doi.org/10.1007/ s00253-003-1240-8

Shimazu, M., 2004. A novel technique to inoculate conidia of entomopathogenic fungi and its application for investigation of susceptibility of the Japanese pine sawyer, $M$. alternatus, to Beauveria bassiana. Applied Entomology and Zoology, vol. 39, no. 3, pp. 485-490. https://doi.org/10.1303/aez.2004.485

Shimazu, M., Sato, H., 2003. Effects of larval age on mortality of Monochamus alternatus Hope (Coleoptera: Cerambycidae) after application of nonwoven fabric strips with Beauveria bassiana. Applied Entomology and Zoology, vol. 38, no. 1, pp. 1-5. https:// doi.org/10.1303/aez.2003.1

Shimazu, M., Tsuchiya, D., Sato, H., Kushida, T., 1995. Microbial control of M. alternatus by application of Nonwoven fabric strips with B. bassiana on infested tree trunks. Applied Entomology and Zoology, vol. 30, pp. 207-2013. https://doi.org/10.1303/ aez.30.207 
Sousa, E., Bravo, M.A., Pires, J., Naves, P., Penas, A.C., Bonifácio, L., Mota, M., 2001. Bursaphelenchus xylophilus (Nematoda: Aphelenchoididae) associated with Monochamus galloprovincialis (Col.: Cerambycidae) in Portugal. Nematology, vol. 3, no. 1, pp. 89-91. https://doi.org/10.1163/156854101300106937 